\title{
EDITORIAL
}

\section{FOOD, GLORIOUS FOOD?}

\author{
Appreciating the importance of microorganisms and microbial effects is crucial to sustaining \\ and improving food production, quality and safety.
}

An abundant and safe food supply is the minimum expectation from our agricultural and food industry. Fulfilling this expectation is a complex process involving plant cultivation, animal husbandry and natural-resource management as well as many different downstream processing steps. At each stage, the microbial world has a major impact - both positive and negative.

Exploiting the beneficial properties of microorganisms in the production of food is an ancient art. Early societies discovered that bacteria and fungi, through fermentation processes, could be used to maintain a safe, plentiful and long-lasting supply of foodstuffs such as cheese. Thousands of years later, we now ingest live microorganisms directly, in the expectation that they will confer beneficial physiological effects through specific microbial actions. Even at the most fundamental biological level, microorganisms can directly impact on, and benefit, agricultural plants and animals. A classic example of mutualism in action is the partnership between Rhizobium and legumes, in which the bacteria fix nitrogen into a form that is exploitable by the plants.

The detrimental effects of microbial life on agricultural productivity are equally well documented. Disease-causing microorganisms continually challenge the animals and crops that society relies on for food, and the constant spread and evolution of agricultural pathogens is a growing problem for product yield and consumer safety. Because of the enormous difficulties in eradicating microbial contaminants from agricultural goods, microbial interference in the food chain continues long after the food has left the farm. Food contamination creates a massive social and economic burden on global health systems. In the USA, approximately 76 million cases of food-borne diseases are estimated to occur each year, resulting in 32,5000 hospitalizations and 5,000 deaths. Diseases caused by the major food pathogens alone are estimated to cost up to US\$35 billion annually in medical expenses and lost productivity.

The microbial world, however, is not just a source of hardship for the food and agriculture industry. Many of the solutions to microbial disease and spoilage lie in the successful application of microbiology itself. To ponder on these issues and also examine the future of food and agriculture microbiology, the American Academy of Microbiology (AAM) recently convened a gathering of leading experts in these fields. As highlighted in their subsequent report, a more complete understanding of the biology of the microorganisms causing disease and spoilage in food will be essential, not only to control these organisms, but also to maximize the potential of beneficial microorganisms. In situations where plant and animal infectious diseases cannot be eliminated, effective strategies for rapid containment and destruction are required. As disease spreads, the resources that are required for adequate quarantining and culling come under tremendous pressure, as was clearly illustrated by the 2001 outbreak of footand-mouth disease in the UK and the ongoing outbreaks of avian influenza. Improved surveillance of, and response to, outbreaks of disease in plants and animals is also crucial, a capability that requires a much greater knowledge of microbial communities than is currently available.

The AAM report also highlights the barriers that must be overcome if the full potential of food and agriculture microbiology is to be realized. Financial support for research in this field has decreased significantly in recent years, and the lagging priority of food and agriculture microbiology within both funding organizations and research institutions must be addressed if these trends are to be reversed. Worryingly, the decline in funding has also been accompanied by a decline in the number of young scientists entering the field. Clearly, raising the profile of food and agriculture microbiology and building awareness of the intrinsic value of an active research agenda is crucial. As one small contribution towards that goal, Nature Reviews Microbiology has recently initiated a new article series aiming to provide an authoritative overview of key areas in the field of food microbiology.

Ultimately, progress in communicating the importance of a strong food and agriculture research agenda to key decision makers, and the revitalization of the field, will require the shared commitment of microbiology-focused organizations, societies and, indeed, microbiologists themselves. The AAM report is a welcome first step. 